FACULDADE DE CIÊNCIAS ECONÔMICAS DA UFRGS
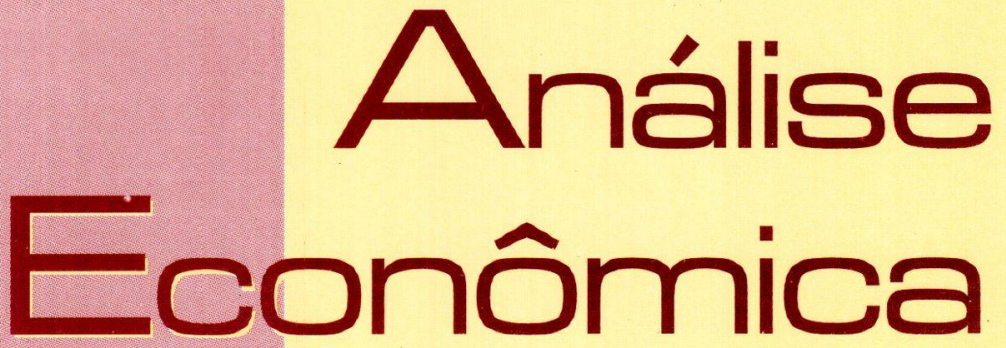

AS LOCOMOTIVAS ENFERRUIADAS

DO CAPITALISMO

Reinaldo Gonçalves

EFICIENNCIA, DESRECULAMENTACC̃O

FINANCEIRA E CRESCIMENTO

ECONOMICO: UMA ABORDAGEM

PÓS-KEYNESIANA

ROGÉRIO SOBREIRA

MERCADOS CONTESTÁVEIS E

COMPEIIÇÃO NO NOVO MODELO

DO SETOR ELÉtRICO BRASILEIRO

ELBIA VINHAES

EDVALDO SANTANa

MICROCRÉDITO: ASPECTOS TEÓRICOS

E EXPERIÊNCIAS

Márcio BobiK Braga

RUDINEI TONETO JR.

A RelaÇão Entre a Política e A ECONOMIA: SUAS IMPLICAÇÓES NO SISTEMA FINANCEIRO

JORGe PESSOA De MENDONĢA

AS RAiZzes CONCEITUAIS DO

INERCIALISMO

VAIDIR RAMAIHO

AUGE E INICIO DO DECLINIIO DA ECONOMIA ARGENTINA

maria Heló́sa lenz

$$
\text { ANO } \begin{array}{r}
18 \\
\text { No } 33
\end{array}
$$

MARÇO, 2000 
Universidade Federal do Rro Grande do Sui

Reitora: Prof ${ }^{a}$. Wrana Maria Panizzi

Faculdade de Ciências Econômicas

Diretora: Prof ${ }^{a}$ Otilia Beatriz Kroeff Carrion

Centro de Estudos e Pesquisas Econômicas

Diretor: Prof. Fernando Ferrari Filho

Departamento de Ciênctás Econòmicas

Chefe: Prof Luiz Alberto Oliveira Ribeiro de Miranda

Curso de Pós-Graduação em Economia

Coordenador: Prof. Marcelo Savino Portugal

Curso de Pós-Graduação em Economia Rural

Coordenador: Prof. Carlos Guilherme A. Mielitz Netto

Consel.ho Editorial:

Achyles B. Costa, Aray M. Feldens, Carlos A. Crusius, Carlos G. A. Mielitz Netto, Eduardo A. Maldonado Filho, Eduardo P. Ribeiro, Eugênio Lagemann, Fernando Ferrari Filho, Gentil Corazza, Marcelo S. Portugal, Nali I. Souza, Otnia B. K Carrion, Paulo A. Spohr, Paulo D. Waquil, Pedro C. D. Fonseca, Roberto C Moraes, Ronald Otto Hillbrecht, Stefano

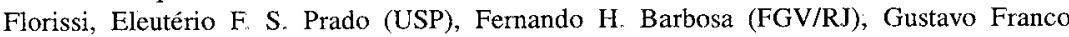
(PUC/RJ), João R. Sanson (UFSC), Joaquim P. Andrade (UnB), Juan H. Moldau (USP), Paul Davidson (Univ. of Tennessee), Werner Baer (Univ. of Illinois).

\section{Comissāo Entrorial:}

Eduardo Augusto Maldonado Filho, Fernando Ferrari Filho, Gentil Corazza, Marcelo Savino Portugal, Paulo Dabdab Waquil; Roberto Camps Moraes.

EorTor: Gentil Corazza

Edrror Adjunto: Pedro Silveira Bandeira

Secretário: Márcio Souza de Vargas

Revisão de Textos: Vanete Ricacheski

Fundador: Prof Antônio Carlos Santos Rosa

Os materiais publicados na revista Análise Econômica são da exclusiva responsabilidade dos autores. É permitida a reprodução total ou parcial dos trabalhos, desde que seja citada a fonte Aceita-se permuta com revistas congêneres. Aceitam-se, também, livros para divulgação, elaboração de resenhas e recensões. Toda correspondência, material para publicação (vide normas na terceira capa), assinaturas e permutas devem ser dirigidos ao seguinte destinatário:

PROF GENTIL CORAZZA

Revista Análise Econômica - Av. João Pessoa, 52

CEP 90040-000 PORTO ALEGRE - RS, BRASIL.

Telefones: (051) 316-3348 e 316-3440 - Fax: (051) 316-3990

E-mail: rae@vortex.ufrgs.br

Análise Econômica

Ano 18, $\mathrm{n}^{\circ} 33$, março, 2000 - Porto Alegre

Faculdade de Ciências Econômicas, UFRGS, 2000

Periodicidade semestral, março e setembro

1. Teoria Econômica - Desenvolvimento Regional -

Economia Agrícola - Pesquisa Teórica e Aplicada -

Periódicos. I. Brasil.

Faculdade de Ciências Econômicas,

Universidade Federal do Rio Grande do Sul. 


\section{As raízes conceituais do inercialismo}

Valdir Ramalho*

Resumo: A tese de que uma elevação geral de preços se perpetua é o núcleo de uma elaborada visão de inflação, o inercialismo. Este artigo levanta as origens dos elementos centrais dessa visão, enfocando trabalhos cruciais de eminentes pesquisadores da inflação entre os inícios dos anos 50 e 80, tais como Franklyn Holzman, James Duesenberry, Gardney Ackley, Charles Schultze, L. Dicks-Mireaux, K. K. F. Zawadzki, Arthur Okun, Georgy Perry, Michael Wachter, Martin Baily, John B. Taylor, James Tobin, e outros. Há aqui uma breve história dos conceitos de choque inflacionário, espiral preços-salários, conflito distributivo, Curva de Phillips, expectativas retrospectivas, contratos justapostos, patamar inflacionário, autoperpetuação da inflação, inércia de preços e salários, e inflação institucionalizada pela indexação.

Palavras-chave: Choque inflacionário, inflação inercial, indexação.

Abstract: The thesis that a general price rise perpetuates itself is the core of inertialism, an elaborate view of inflation. This article traces the origins of the central elements of that view, focusing on crucial works by eminent researchers of inflation from the early 50 s to the early 80 s, such as Franklyn Holzman, James Duesenberry, Gardney Ackley, Charles Schultze, L. DicksMireaux, K. K. F. Zawadzki, Arthur Okun, Georgy Perry, Michael Wachter, Martin Baily, John B. Taylor, James Tobin, and others. Here is a brief history of the concepts of inflationary shock, wage-price spiral, distributive conflict, Phillips Curve, backward-looking expectations, overlapping contracts, inflation plateau, self-perpetuating inflation, price-wage inertia, and inflation institutionalized through indexation.

Key-words: Inflationary shock, inertial inflation, indexation.

\section{Introdução}

Uma inflação alta e crônica é inercial - a alta de preços é simplesmente herdada do passado. Assim é conhecido o diagnóstico do inercialismo, uma visão de inflação que veio a ter grande influência no país por alguns anos, a partir de 1984. A importância que ganhou justifica a tarefa de colocar tal doutrina sob a perspectiva da história das idéias (que em economia se chama história do pensamento econômico).

\footnotetext{
* Professor Universitário e Assessor de Pesquisas em Ciências Sociais e Humanidades.
} 
Os benefícios de conhecer o passado de idéias e sua evolução são os mesmos do estudo da história em outros campos. Em especial, cultivar a história é uma exigência de sociedade civilizada, isto é, daquela formada por gente de espírito refinado. Ao relembrar nomes e obras, mostramo-nos capazes de ser gratos para com aqueles que desenvolveram seus talentos e trabalharam arduamente para legar-nos idéias frutíferas, inspiradoras ou úteis. Pronunciamos para a nossa própria geração os nomes daqueles que construíram para a humanidade, e não somente para si mesmos. E pelo mesmo instrumento, o recontar a história, o citar quem e o quê, punimos aqueles que, por incompetência, descaso, ou má fé, deixaram-nos herança destrutiva. Ademais, a experiência dos nossos antepassados intelectuais ensina-nos a evitar a repetição de erros, bem como a notar ou avaliar melhor obstáculos que foram defrontados por eles primeiro. As idéias que eles desenvolveram são matéria-prima para a nossa própria elaboração de outros produtos intelectuais e tecnológicos.

Este trabalho expõe as concepções sobre inflação que deram origem à visão da inflação inercial. Como se verá, as noções centrais dơ inercialismo remontam a 1950 na literatura econômica anglo-saxônica, e se tornaram muito comuns nos anos 60 e 70; desenvolvimentos e acréscimos foram feitos nos anos 70, época em que paralelamente ocorreu a influência dos trabalhos de Mário Henrique Simonsen no Brasil. Mas a contribuição de Simonsen, por si só, requer o espaço de um artigo, e por isso não será abordada nesta oportunidade.

A título de ilustração, estas são importantes noções incorporadas pelo inercialismo e advindas de décadas anteriores aos anos 80: o conflito distributivo como mecanismo de inflação; a "persistência" ou "repetição" indefinida do processo inflacionário; a explicação dessa característica como resultante do mecanismo de determinação de preços e salários; a caracterização desse mecanismo como sendo, em certos casos, vínculo automático entre ajustes de custos, preços e salários, e em particular como dependente de margem de lucros constante e da defasagem nos ajustes; a capacidade desse processo resultar em perpetuação ou realimentação da inflação; as noções de choque inflacionário e de patamar da inflação; fórmulas em que a taxa de inflação depende da taxa de inflação passada; o papel dos contratos e da indexação na institucionalização e automatização do vínculo mútuo entre preços e salários; a indexação como realimentador da inflação ou como obstáculo à queda da inflação; os termos inércia inflacionária e inflação inercial; e o slogan inercialista de que a inflação passada se reproduz no presente.

Este trabalho está dividido em 6 seções. A primeira, introdução, expõe uma visão bastante conhecida nos anos 60, e que remonta pelo menos a 1950 : há persistência da inflação por causa da dependência mútua entre preços e 
salários, chamada de espiral preços-salários. A segunda seção apresenta procedimentos de estabelecimento de preços e salários que levam a dependência entre preços presentes e passados através de expectativas retrospectivas e de contratos baseados em informações passadas. Em seguida, na seção 3, exibese uma amostra das idéias inercialistas desenvolvidas nos anos 70 por Arthur Okun, George Perry, e outros expoentes que publicavam no prestigiado periódico acadêmico Brookings Papers on Economic Activity. A origem dos termos inércia e seus cognatos em trabalhos norte-americanos é o tema da seção 4. Documenta-se na seção 5 a presença de oposição à indexação na literatura internacional dos anos 70. Conclui-se o trabalho na seção 6 , chamando-se a atenção para duas variantes da visão de inflação inercial.

\section{Espiral preços-salários}

É concebível a hipótese de que todo processo inflacionário tende a resultar imediatamente e por si mesmo em firme e definitiva aceleração explosiva ou, alternativamente, em rápida tendência a se extinguir. Aparentemente, contudo, não há na história das idéias sobre inflação quem tenha sustentado tal proposição. A noção comum é de que, na grande maioria das vezes, um processo inflacionário dura um considerável período de tempo. As análises monetárias da inflação freqüentemente mencionaram a "defasagem" de mudanças monetárias no efeito sobre preços e sobre a atividade econômica para indicar isso. Mais clara ainda é a noção corrente na literatura dos anos 60 e 70 e indicada com termos como "espiral sem fim", ou "cadeia sem fim", de alta de preços (Ackley, 1961, p. 453, 454; Keiser, 1971, p. 333; Blyth, 1977, p. 54), processo inflacionário "contínuo" (Crouch, 1972, p. 251, 282), com "repetição", ou "persistência" indefinida (Rowan, 1974, p. 466, 467), "espiral inflacionária" ou "círculo vicioso da inflação" (Guitton, 1974, p. 431, 434). Uma expressão muito comum da época era a "espiral preços-salários", que designava a hipótese mais detalhada de como aquela persistência ocorria. É desnecessário enfatizar que a noção e expressões semelhantes continuaram populares até pelo menos o início dos anos 80. O tema do artigo de Gylfason e Lindbeck (1982), por exemplo, é exatamente a mencionada espiral (p. 431), isto é, um “... vicious circle of wage and price increases..." (p. 434, 438, 444).

A concepção da espiral era de que aumentos no nível geral de preços levam a aumentos nos salários, e aumentos de salários resultam em aumentos de preços (Holzman, 1950; Schultze, 1959, p. 372; Dicks-Mireaux, 1961; Ackley, 1961, p. 452-459; Johnson, 1963, p. 109; Keiser, 1971, p. 333; Rowan, 1974, p. 470; Nordhaus, 1976, p. 60-61; Wachter, 1976, p. 69; 
Blyth, 1977, p. 51-52, 84, Venieris e Sebold, 1977, p. 613; Hagger, 1977, p. 30-32). Keynes foi um dos primeiros a empregar essa concepção nos anos 40 para explicar o fenômeno inflacionário, conforme Trevithick e Mulvey (1975, p. 18-19). Assim, um aumento inicial em um desses dois fatores, preços ou salários, poderia levar a aumento no outro e a subseqüente reação do primeiro fator, e assim indefinidamente. Em princípio, a idéia da espiral é compatível com qualquer teoria da inflação; contudo, recorreram a ela sobretudo os proponentes de três teorias: a de custos, a de margens ('markup'), e a de conflito distributivo, todas extensamente desenvolvidas dos anos 40 aos anos 60 (ver Zawadzki, 1965; 1975). A razão disso é óbvia; essas teorias dependem muito mais de especificação do mecanismo de propagação da inflação do que a teoria da demanda e a teoria monetária.

$\mathrm{O}$ artigo clássico que formalizou e divulgou a hipótese do conflito entre segmentos da sociedade pela participação na renda agregada como base do processo inflacionário é de autoria de Franklyn Holzman (1950). Este também foi o primeiro a definir e introduzir na literatura o conceito "inflationary schock" (p. 151; há amplo uso deste em Zawadski, 1965; 1974, p. 41). Em passagem que resume sua posição, Holzman fez um contraste com a teoria dominante da época, a do multiplicador keynesiano: "Resistance by economic groups to loss of real income which comes about through differential costprice rises, rather than the multiplier process of the Keynesian approach, is the dynamic element in the inflationary process." (p. 150)

Na mesma época, surgiu outro trabalho importante sobre o tema, o de James Duesenberry (1950). Para este, a inflação resulta da interação entre preços industriais determinados por margem de lucros e salários determinados por mudanças no nível de preços. Duesenberry supôs que os preços agrícolas são determinados competitivamente no mercado, o que faz sua explicação ser um importante antepassado da geração de modelos com dois setores que proliferou nos anos 70 . E ele foi talvez o primeiro a afirmar que a inflação poderia subsistir na ausência de excesso de demanda: "In our system, the speed of inflation is governed by the lags in wage adjustments and by the other factors which control the rate of agricultural prices. The inflation need not stop when excess demand is eliminated." (p. 149)

A hipótese da rivalidade pela participação na renda e a hipótese da margem de lucros calculada sobre custos deram origem a uma literatura crescente nos anos 50 e 60 e se popularizaram em livros didáticos de macroeconomia e livros sobre inflação, tais como o de K. K. F. Zawadzki $(1965 ; 1975)$. A título de ilustração, vejamos algumas passagens desse autor expondo a visão do conflito distributivo e publicadas nos anos 60 : 
... la causa de la subida de precios no está en el exceso de demanda, sino en la decisión de los empresarios de mantener sus márgenes de beneficios ante la amenaza de la subida de los salarios reales de los obreros. $(1974, p .12)$

... la inflación se origina por la tentativa de un grupo social de alterar a su favor la distribución de la renta real y a expensas de otro grupo o grupos capazes y dispuestos a emprender reacciones defensivas. (1974, p. 14)

Zawadzki também enfatizou a noção de continuidade ou persistência desse tipo de inflação:

La característica más importante de este tipo de inflación es su automatismo. Cada variación de los precios, autónoma o inducida, originará una variación en la misma dirección del nivel de salarios; cada variación de los salarios, autónoma o inducida, causará una variación en la misma dirección del nivel de precios. $(1974$, p. 18)

Los procesos de este tipo, una vez iniciados, continuarán en tanto que el método de determinar los precios con respecto a los costes y el sistema de reajustar los salarios a los cambios en el nivel de precios funcionen. (1974, p. 19)

Si los salarios se llevan siempre en la misma proporción que los precios, y si los precios se elevan en la misma proporción que los salarios, la inflación de este tipo continuará indefinidamente. (1974, p. 22)

Note-se o uso do termo "autônomo", comum na literatura keynesiana da época, com o significado de choque inflacionário (1974, p. 41). Pode-se ainda obter um bom quadro da discussão sobre hipótese do conflito distributivo, mais de 30 anos depois, em um retrospecto favorável à mesma e escrito por Gylfason e Lindbeck (1982).

O fato é que, em maior ou menor grau, as teorias de inflação de custos, de margens e de conflito distributivo deram abrigo à visão da espiral inflacionária. Segundo esta, o que gera a "cadeia sem fim" de alta de preços é a tentativa de elevar ou manter remuneração real, quer seja por trabalhadores, quer seja por empresas (Holzman, 1950; Ackley, 1961, p. 454; Johnson, 1963 , p. 117 ; Blyth, 1977 , p. 52 , 54, 55; Makinen, 1977, p. 341). Obviamente, a base desse processo é formada pelos procedimentos de estabelecer preços e os procedimentos de estabelecer salários (Holzman, 1950; DicksMireaux, 1961; Ackley, 1961, p. 458; Tobin, 1974, p. 321; Rowan, 1974, p. 470-471, 473-474; Blyth, 1977, p. 52, 53, 55). De particular importância é a 
defasagem na reação (Dicks-Mireaux, 1961,-Zawadzki, 1965; 1974, p. 30; Rowan, 1974, p. 469. 571; Blyth, p. 54; Venieris e Sebold, 1977, p. 614; Hagger, 1977, p. 31); os aumentos de salários levam em conta, não a inflação do período em que irão vigorar, mas a inflação de um período anterior. Do mesmo modo, os aumentos de preços são determinados por aumentos anteriores de salários, e não por aumentos que ocorrerão no período de vigência dos novos preços.

Charles Schultze, em artigo clássico (1959), resumiu esse processo assim: "If prices are set by applying a constant margin to costs, and if wages are determined by movements in the level of consumer prices, then an initial general price rise, stemming from any source, can perpetuate itself, as wages and prices successively adjust upward to each other." (p. 372).

Nesta passagem, é particularmente digna de nota uma referência pioneira à capacidade da inflação de se perpetuar, noção que veio a ser tão enfatizada no Brasil dos anos 80 . Ademais, é óbvio que o texto diz respeito a um processo em que aumentos de preços geram aumentos de preços, intermediados por aumentos de salários. Além do efeito de salários sobre preços, os economistas dos anos 60 e 70 reconheciam que os aumentos de preços levavam diretamente a aumentos de preços dos materiais e insumos (Ackley, 1961, p. 453; Keiser, 1971, p. 333). Alguns economistas da época deduziram explicitamente fórmulas em que a taxa de inflação depende da taxa de inflação passada, quer exclusivamente, quer parcialmente. Este é o caso do artigo clássico de Dicks-Mireaux (1961), em que preços dependem de preços passados através de salários, bem como de Rowan (1974, p. 471, 472). Fórmula semelhante é reproduzida por Turvey (1978, p. 191). É instrutivo constatar que Trevithick e Mulvey (1975) atribuem uma fórmula desse tipo a Keynes nos anos 40 (p. 19); a J. C. R. Dow em 1956, a L. Klein e R. J. Ball em 1959, bem como a A. G. Hines em 1969 (p. 79); e ainda a M. Kalecki em 1954, bem como a J. Williamson em 1967 (p. 80). Ademais, Harry Johnson (1963), em um famoso retrospecto de teorias de inflação, já havia reproduzido uma dessas fórmulas. $\mathrm{O}$ que ele disse a respeito de vários modelos da espiral inflacionária desde os anos 40 deixa bem claro a característica destes: "At the formal level they involved a first- or second-order difference equation relating the price level or change in the price level in one period to the price level or change in the price level in the previous period or two periods..." (p. 119).

Em termos semelhantes, Trevithick e Mulvey $(1975$, p. 22) fizeram menção a equações de diferenças finitas.

Assim, o nome "espiral preços-salários" obviamente provinha do principal mecanismo de um processo que todos entendiam como sendo também uma "espiral preços-preços". Aliás, em um artigo para o New York Times, 
James Tobin escolheu a expressão "wage-price-wage spiral" (1974, p. 320, 321). Por isso, se ouvissem o slogan inercialista de que a inflação de hoje é determinada pela inflação de ontem, provavelmente Ackley e os economistas dos anos 60 e 70 não veriam nele qualquer novidade. Note-se como o jogo com os termos "ontem" e "hoje" na seguinte citação de Ackley (1961) assemelha-se ao do mencionado slogan: "... not only do prices follow wages but... wages also follow prices. Today's wage increase may seem to require tomorrow's price increase. But, to labor, today's wage increase is often seen as an important measure required by yesterday's increase in the cost-ofliving." (p. 452)

De modo similar, Jerry Pohlman (1976) falou de "inflation as the cause of inflation" (p. 132) e resumiu assim sua análise do fenômeno: "Thus, the inflationary spiral develops an inner logic and momentum of its own and can continue in spite of the fact that there may no longer be demand-pull elements present in the economy." (p. 135).

A expressão momentum é bastante usada na literatura dos anos 70 com referência à inflação, e tem mais ou menos o mesmo sentido com que passou a ser empregado, depois, o termo inércia. O momentum of its own é um impulso intrínseco do processo inflacionário, que se mantém sem ser empurrado por outras forças.

Venieris e Sebold (1977) também enunciaram o slogan inercialista: "The heart of inflation is the dependence of this year's general price increase on those taking place in the recent past." (p. 613)

Note-se também como a implicação prática retirada por Blyth (1977) coincide com as prescrições inercialistas: “... a way to insulate against inflation, or to reduce the propensity to inflate, would be to change price and wage behaviour so as to break the automatic links between cost increases on the one hand and price and wage increases on the other." (p. 55)

E quando Blyth (1977) resumiu a teoria da espiral, a conotação inercialista foi flagrante: "Modern inflation can feed on itself, in the ways described in Chapter 3..." (p. 86).

\section{Expectativas e contratos retrospectivos}

Durante os anos 70, desenvolveu-se uma enorme literatura buscando elaborar os fundamentos microeconômicos da Curva de Phillips. A maior parte dos trabalhos nessa área passou a focalizar o mercado de trabalho, a dinâmica dos salários e a dinâmica dos preços (um exemplo clássico desses esforços é Phelps, et alli, 1970, orgs.). A hipótese da curva de Phillips acabou servindo como elo de ligação entre dois grupos de visões do fenô- 
meno inflacionário: teorias de inflação de demanda, por um lado, e teorias de custos, margens e conflito distributivo, por outro. Este elo proporcionou uma base teórica para modelos que sintetizaram os dois grupos de visões. Isto não quer dizer que, antes dos anos 70, inexistissem modelos representando a inflação como dependente simultaneamente de expansão da demanda agregada e de mecanismos preços-salários; quer dizer que, antes da curva de Phillips, tais modelos faziam uma síntese de modo ad hoc. Alguns exemplos de equações de inflação que incluíam a influência de preços ou salários de um período sobre preços de período posterior, como conseqüência de alguma formulação da curva de Phillips, podem ser vistos em Lemgruber (1977, p. 402, 440).

Um dos usos imediatos e mais importantes da curva de Phillips foi o de introduzir nos modelos macroeconômicos uma representação do efeito de redução da atividade econômica que uma política antiinflacionária provoca. Isso levou a sugestões de como reduzir o grau de conflito entre desemprego e inflação, representado pela inclinação da curva, de modo a fazer com que a curva de Phillips tenha uma inclinação mais favorável aos responsáveis pela política econômica. Uma sugestão chamou a atenção para a ausência de informação e para outras fricções que podem afetar os mercados de trabalho e de produtos, propondo mudanças para aperfeiçoar o desempenho dos mercados, por exemplo, maior acesso a informações sobre vagas de empregos, programas de relocalização de trabalhadores e retreinamento, etc. Outra sugestão foi a de empregar-se controles de preços (políticas de rendas), que supostamente permitiriam a queda da inflação sem redução da atividade econômica.

Paralelamente surgiu uma literatura para explicar a ocorrência do trade-off com base na existência de contratos que fixam salários ou preços por algum tempo. Uma lição óbvia desse enfoque é que o trade-off poderia ser reduzido por meio de medidas que diminuíssem a rigidez dos contratos. Este é um dos mais velhos precedentes na literatura da proposta inercialista de que a redução da inflação teria um alto custo em termos de desemprego, a menos que se desmantelassem os contratos com indexação.

Ramificações da literatura sobre a curva de Phillips expuseram uma explicação um pouco diferente para a dependência de preços a preços, ou seja, para o que Venieris e Sebold (1977) chamaram "The feedback effect of a change in the price level" (p. 616; ver também p. 636). De acordo com essa visão, o mecanismo de realimentação consiste no seguinte (Venieris e Sebold, 1977, p. 617, 623): i) os procedimentos de estabelecimento de preços e salários levam em conta expectativas de inflação; ii) em geral, os agentes econômicos formam tais expectativas a partir da experiência inflaci- 
onária do passado recente (hipótese de expectativas adaptativas). Como seria de esperar, esses economistas também deduzem equações em que a taxa de inflação de um período depende da taxa de inflação passada (por exemplo, Venieris e Sebold, 1977, p. 624).

Toda a literatura levantando as bases microeconômicas da curva de Phillips, antes mesmo da aceitação da tese aceleracionista (a curva de Philllips vertical a longo prazo), pode ser resumida por uma equação desse tipo. Nesta primeira fase da história da famosa curva, as bases microeconômicas eram buscadas em investigações do processo de formação da taxa salarial ou do mercado de trabalho ou da atuação dos sindicatos. Em virtude dos salários receberem compensação ao menos parcial pela inflação passa$\mathrm{da}$, a curva de Phillips pode ser formulada assim (ver Trevithick e Mulvey, 1975, p. 72):

$$
W(t)=f[U(t)]+a \cdot P(t-1)
$$

Esta equação representa uma curva de Phillips do início dos anos 60 , do tipo proposto por R. G. Lipsey. Para os períodos de tempo t e $t-1$, o crescimento percentual da taxa de salários é indicado por $\mathrm{W}$, a taxa de desemprego por $\mathrm{U}$, e a taxa de inflação por P. Uma função $f$ tem derivada primeira negativa.

Acrescentando a hipótese de margem de lucros como uma constante $\mathrm{m}$, $\mathrm{P}(\mathrm{t})=\mathrm{m} \cdot \mathrm{W}(\mathrm{t})$

Assim, conclui-se que

$$
P(t)=m \cdot f[U(t)]+m \cdot a \cdot P(t-1)
$$

Na segunda metade dos anos 60 , a curva de Phillips foi reformulada em função da crítica aceleracionista, introduzindo-se variável representativa das expectativas de inflação. Uma implicação da visão aceleracionista é de que pode ocorrer uma inflação inercial, quando a economia está operando na taxa natural de desemprego:

Inflation can still occur at this rate, depending upon the state of price expectations, but it will be taking place at a constant rate. (itálico no original; Trevithick e Mulvey, 1975, p. 109).

... if the 12 percent inflation rate becomes fully anticipated, the economy can be described as being in a moving stable equilibrium with unemployment at its natural rate. It is deviations in the rate of inflation that cause problems. (Makinen, 1977, p. 362) 
Isso pode ser formalizado da maneira mais simples possível, da seguinte forma (ver Trevithick e Mulvey, 1975, p. 110). Tome-se uma equação da curva de Phillips aceleracionista, onde $\mathrm{Pe}(\mathrm{t})$ indica a taxá de inflação esperada:

$P(t)-P e(t)=f[U(t)]$

Adicione-se a hipótese de que a taxa de inflação esperada é igual à taxa de inflação observada recentemente, ou de modo mais geral, é igual a uma função das taxas de inflação vivenciadas nos $\mathrm{n}$ períodos do passado que influenciam os agentes econômicos:

$\operatorname{Pe}(\mathrm{t})=\mathrm{h}[\mathrm{P}(\mathrm{t}-1), \mathrm{P}(\mathrm{t}-2), \ldots, \mathrm{P}(\mathrm{t}-\mathrm{n})]$

Conseqüentemente,

$\mathrm{P}(\mathrm{t})=\mathrm{f}[\mathrm{U}(\mathrm{t})]+\mathrm{h}[\mathrm{P}(\mathrm{t}-1), \mathrm{P}(\mathrm{t}-2), \ldots \mathrm{P}(\mathrm{t}-\mathrm{n})]$

Uma equação semelhante foi estimada por R. Solow em 1969 (Trevithick e Mulvey (1975, p. 114), bem como por vários outros economistas (idem, p. 115; ver também Hagger, 1977, p. 138). Como outro exemplo, Lucas e Rapping (1969) procuram mostrar que o efeito da inflação no nível de atividade econômica descrito pela curva de Phillips é um fenômeno de curto prazo. Com esse fim, estimam uma equação que embute a hipótese de que os preços dependem de preços passados (p. 345).

A análise dos mecanismos de dependência de preços a preços entrou em uma nova fase com a introdução de nova versão da hipótese de que muitos preços estão amarrados a dispositivos contratuais. Uma das versões é a hipótese de contratos justapostos ou escalonados, cuja melhor formalização foi feita por John B. Taylor $(1979 ; 1980)$, em um modelo simples, mas original e instigante, com base nos seguintes elementos: i) no que concerne uma parcela grande dos preços e salários na economia, eles são determinados em contratos; ii) os diversos contratos vencem em épocas diferentes, de modo que a cada momento somente uma pequena fração dos contratos existentes compõe-se de contratos sendo negociados ou revisados; iii) cada contrato embute uma estimativa de inflação com base nas informações disponíveis na época em que é celebrado. Em consequência disso, iv) a cada momento os reajustes de preços e salários são, na maior parte, predeterminados por contratos que estão em vigência; v) os contratos ainda não vencidos basearam-se em informações atualizadas na época em que foram assinados; mas como estas datas agora pertencem ao passado, os reajustes de preços e salários que ocorrem neste período se baseiam em informações passadas de inflação.

A conclusão é que a existência de contratos gera persistência de inflação, pois a taxa de inflação de cada período é, em grande parte, predetermi- 
nada por taxas de inflação observadas ou previstas no passado. Sucintamente, Gylfason e Lindbeck (1982) expressam essa conclusão assim: “... overlapping contracts contribute to price and wage inertia..." (p. 443).

Uma análise bastante similar foi feita por Olivier Blanchard (1983), que explicou a inércia dos preços com base na falta de sincronização das decisões de ajustar preços. Seu trabalho foi divulgado em 1981 em uma conferência no Rio de Janeiro sobre inflação e indexação. Paralelamente, em seu livro de macroeconomia (1981), Arthur Okun também enfatizou o papel de contratos dessincronizados na determinação da evolução de preços e salários a curto prazo.

\section{Okun e a equipe do brookings papers}

A influente publicação acadêmica Brookings Papers on Economic Activity abrigou durante os anos 70 um pequeno grupo que desenvolveu ativamente a visão da inflação como um fenômeno inercial: Arthur Okun, George Perry, James Pierce e Jared Enzler, Michael Wachter, Martin Baily.

George Perry (1971) contribuiu para a divulgação da noção de perpetuação da inflação, usando o termo "habitual" referindo-se ao que mais tarde seria chamado de inércia: "... I am persuaded that inflation is now perpetuated to an important degree because of high "habitual" rates of wage and price increase" (p. 446).

Em artigo seguinte, Perry (1972) esclareceu seu ponto de vista com base em duas equações. Na primeira, o reajuste de salário de um período é determinado pela inflação do período anterior, com um parâmetro q de reação dos salários a preços; a segunda representa o comportamento das firmas e mostra preços dependendo de uma margem sobre salários, com o parâmetro $r$ medindo os repasses de custos a preços: Delas deduz uma terceira equação em que a taxa de inflação de um período é proporcional à inflação do período anterior. E conclui:

"[if] $\mathrm{r}=\mathrm{q}=1$, inflation will not slow down." (p. 192)

Arthur Okun (1975) construiu um modelo em que relações contratuais implícitas ou explícitas entre vendedores e compradores ou entre patrões e empregados fazem com que, em parte da economia, preços e salários dependam de preços e salários passados. $O$ resultado é o fenômeno da inércia inflacionária: "Inflation starts slowly but builds up a momentum that keeps its going even after its initiating source is eliminated." (p. 378)

Okun descreveu assim o que ocorre quando as instituições passam a se adaptar à inflação mais alta: "Most of these adaptations shorten the lags and increase the intensity of inflation associated with any given level of real 
economic activity. Thus, they generate an adverse shift of the short-run Phillips curve that reflects the inflationary experience of the past." (p. 382).

Em outro trabalho, Perry (1975) fez uma resenha de estimativas de equações e de experiências inflacionárias em que há interação entre preços e salários. A certa altura, explicou: “... the prominence of either lagged wages or inflations in the wage equations shows that once a rate of wage increase is established, there is a good deal of inertia acting to perpetuate it." (p. 432)

Ligada à noção de inércia estava a de patamar inflacionário: "... the inertia in wage inflation... helps explain why rates of wage increase stayed on a higher plateau after their year of explosion." (p. 432)

As conclusões de Perry foram que: [i] “... a great deal of inertia characterizes wage inflation in most countries;" [ii] "... conflict over income shares has been a source of wage inflation... " (p. 434); [iii] "... because the inertia of inflation is strong, slowing inflation with higher unemployment will be very costly." (p. 435)

Michael Wachter (1976) considerou o que ele caracterizou como “... the popular view, espoused in the academic literature and the financial press, that wages are becoming less sensitive to aggregate demand policies." (p. 158). Conforme tal visão, salários são determinados por preços e preços regem custos, de modo que "What emerges in this context is an autoregressive system in which wages (and prices) follow some combination of their own lagged values." (p. 118).

$\mathrm{E}$ ele achava que a adaptação institucional a inflação mais rápida aumenta a sensibilidade da economia à demanda agregada (p. 125).

Martin Baily (1976) empregou a teoria dos contratos, cuja literatura crescera desde o início dos anos 70 , para focalizar a persistência ou continuidade da inflação por causa da interação entre preços e salários (p. 585, 587). Ele chamou a inércia de "inflationary momentum" (p. 611), que depende da freqüência e da duração dos contratos de salários. Baily mostrou que "... in such a world, it is possible, at least in principle, to design a "request-control" system of wage and price controls that would slow inflation without the loss of real output resulting from demand-restraint policies..." (p. 587).

Em outro artigo, Perry (1978) construiu um modelo de mútua causalidade entre preços e salários para explicar a inércia inflacionária (p. 262). A respeito do comportamento dos salários, ele disse:

The existence of large lagged effects created inertia in inflation that transmitted past inflation to current wage changes even when current unemployment rose. In addition, the importance of these lagged effects 
apparently grew as inflation itself became more entranched. Today inertia is a more important characteristic of the inflation process than it was in the 1950s and 1960s. (p. 278). (p. 278).

Em resumo, a seu ver, “... inertia is a backward-looking phenomenon.”

Okun (1980) voltou a focalizar contratos implícitos no mercado de trabalho $\mathrm{e}$ "... the inflationary inertia that is now built into our implicit contracts." (p. 11). Ele afirmou que tais contratos afetam salários e preços e, por isso, a inflação cai pouco e lentamente quando a demanda agregada é reduzida (p. 8). E prosseguiu: "In general, as people adjust to an inflationary world, they make inflation more rapid and more persistent. Any prolonged experience with an inflation rate well above the secular average to which the system has become adapted alters implicit and explicit contracts in ways that make the inflation feed upon itself." (p. 9).

A idéia de inércia inflacionária foi também explorada por Otto Eckstein (1981), que preferiu verbalizá-la como "inflação-núcleo" ("core inflation").

\section{Termo inércia e cognatos}

Embora seja difícil encontrar sua origem precisa, dois termos interrelacionados da física, impulso ("momentum") e inércia (e vocábulos derivados), tornaram-se bastante usados, a partir dos anos 70, para expressar a persistência ou continuidade do processo inflacionário. O termo "impulso" abreviava a noção de que a inflação prosseguia por seu próprio impulso. Perry $(1975$, p. $410,432,434)$ parece ter sido o primeiro a usar o termo inércia para indicar o efeito de preços passados ou outras condições do mercado de trabalho sobre os preços presentes. Logo, o termo voltou a ser usado por' Wachter $(1976$, p. 69,70$)$ e de novo por Perry $(1978$, p. 278$)$. Depois, em artigos que se tornaram muito conhecidos por sua originalidade e pertinência, John B. Taylor $(1979$, p. 110; 1980, p. 1, 3, 21) empregou o vocábulo para expressar a persistência dos reajustes salariais e de preços de um período para outro.

Em seminário sobre inflação realizado em 1979, o termo "inércia" foi empregado com freqüência por Rudiger Dornbusch e Stanley Fischer (1981, p. 326, 329, 331). Os autores descrevem assim um modelo que combina hipóteses de margens de lucros na formação de preços e existência de contratos: "In such a model the current rate of wage inflation will be a weighted average of the predetermined rates of inflation in existing contracts of 
various maturities and of the rate of wage inflation of contracts currently entered into." (p. 325).

E em um conhecido trabalho sobre inflação, divulgado no mesmo seminário e posteriormente publicado em livro, James Tobin (1981, p. 23, 24, $25,27,29)$ usou a expressão "inertial inflation" e deu a seguinte definição:

By 'inertial inflation' I mean the self-replicating pattern of wage and price inflation with which we all have become familiar. Whatever the historical origins of the inflation, once it is built into the system in a. consistent manner it continues very much on its own. (p. 23).

In any case, what matters is the stubborn persistence of the built-in pattern, in particular its resistance to slowdown in monetary demand. Refusal to accommodate inertial inflation will not cause the pattern to collapse. Rather it will bring severe contraction in employment and production for several years, while the built-in wage/price pattern subsides very slowly. (p. 24).

Em artigo sobre tipos de inflação, causas e curas, Tobin (1980) voltou a explicar o que entendia por inflação inercial:

First, inertial inflation, as the name implies, is inherited from the past... Second, inertial inflation is perpetuated by contracts and commitments, particularly with respect to wages. (...) Third, the inertia in wages that results from this system supports and is supported by the mark-up pricing conventions of employing firms. (...) The circle is closed as the price increases feed back into the workers' cost of living, reinforcing the pattern of wage increase itself. (..) Adjustment lags are intrinsic to the contracts, institutions, and conventions of wage and price setting. These provide inertia in the adjustment of wages and prices to economic slack, even when expectations change (p. 327-8)

De modo semelhante, em conferência de 1981, William Nordhaus explicou: "Inflation is taken to be the sum of inertial, cyclical, and volatile or random forces. The inertial element is the inherited "underlying" rate of inflation, particularly from wages, which changes slowly in response to experience and expectations." (1983, p. 254).

Inflação inercial tornou-se um tema comum em Tobin (por exemplo, ele voltou a mencioná-la ao escrever sobre o verbete "inflação" para uma enciclopédia; 1982, p. 312-313). E o uso da expressão ou outra semelhante 
também foi adotado por Robert Hall, George Perry, Robert Gordon, Michael Spence e William Nordhaus (ver seus trabalhos em conferência de 1981 publicada em Tobin, org., 1983).

Outros exemplos do emprego do termo no início dos anos 80 são Okun (1980, p. 11), bem como Olivier Blanchard (1983) em trabalho apresentado no Rio de Janeiro em, 1981. Vale a pena citar ainda o artigo de Gylfason e Lindbeck (1982) resenhando inflação de custos, que fala de "price inertia inflation" (p. 441) e "price-wage inertia" (p. 442, 443).

\section{Oposição à indexação}

Nos Estados Unidos no início dos anos 70, o dispositivo da indexação era quase desconhecido pelos economistas. Mesmo assim, algumas especulações dessa época já tinham conduzido a proposições de que a indexação de salários poderia gerar uma inflação persistente. Em um livro-texto de macroeconomia, John Lindauer (1971) alude a tais proposições: "Other economists... foresee almost immediate new cost increases if prices are allowed to increase since money wages often are tied to the price level by cost-of-living indexes; they fear a continual "wage-price inflationary spiral"..." (p. 347.348)

Poucos anos depois, a inflação alta nos países desenvolvidos criou ambiente propício para que se introduzissem propostas de indexação nos debates de política econômica. Imediatamente apareceram economistas contrários, quase sempre brandindo teses como a de que a indexação estabilizaria a taxa de inflação, ou de que impediria o combate à inflação. Aludindo a esse debate, o Economic Report of the President de 1975 (United States Government, 1975) diz que há diferenças de opinião entre os economistas (p. 141) sobre a questão se a indexação impediria ou retardaria um processo de queda da inflação. Mencionando "the automatic response of indexed wages to price deceleration, and the feedback of that response on prices", acrescenta: "On the other hand, the view has been expressed that in past phases of accelerating inflation such automatic responses would have further steepened the inflation rate and that they also might be damaging in future phases of inflationary processes." (p. 141-142).

Essa visão foi compartilhada por muitos profissionais. Okun (1975) mostrou-se contrário à indexação, temendo sua generalização: "If everybody's price is geared to full escalation with a short lag, the inconsistency of real aims must result in explosive inflation." (p. 386)

De modo semelhante, Blyth (1977), um dos primeiros a considerar uma economia indexada dentro da teoria da espiral, diz: “... indexation will cau- 
se, in time, the rate of inflation to settle down to the average rate of increase of those prices not covered by the indexation control scheme..." (p. 80).

Venieris e Sebold (1977) também fazem breve referência à indexação: "... the dependence of wages on prices is often institutionalized in contractual agreements through the stipulation of escalator clauses." (p. 614).

Encontram-se referências adicionais a posições pessimistas sobre indexação, ou a literatura sobre tais posições, em Meade (1975, p. 22-23), Venieris e Sebold (1977, p. 644, 645), Patinkin (1979, p. 132), e Sobel (1979, p. 274). Naturalmente, a literatura do início dos anos 80 continuou aludindo a este tipo de problema que a indexação traria (Gylfason e Lindbeck, 1982):

... indexation insulates real wages from changes in productivity, terms of trade, and so on, and thus tend to accentuate cost inflation when unhindered market forces would otherwise contribute do price stability. Indexation is especially dangerous when introduced under unfavorable initial conditions such as when the sum of the claims on national income exceeds one hundred percent, for under such circumstances indexation locks the economy into a vicious circle of wage and price increases from which there can be no acceptable exit other than the abolition of indexing. (p. 444).

Publicações internacionais que analisam a experiência brasileira também aludem a críticas de que o dispositivo institucionaliza ou realimenta a alta de preços (por exemplo, Baer, 1979, p. 178). Provavelmente elas ecoam o próprio movimento brasileiro de oposição à correção monetária, surgido no início dos anos 70 e liderado por Mário Henrique Simonsen. Em um livro sobre o dispositivo, publicado em 1977, Walter L. Ness Jr. menciona a existência das críticas e esboça o teor de algumas delas: “... muitos efeitos da correção monetária tendem a manter a taxa de inflação existente..." (p. 111). "... quanto maior a extensão da indexação na economia, maior a dificuldade de aplicação de freios ao processo inflacionário." (p. 113).

\section{Conclusão}

A literatura que originou e desenvolveu as noções fundamentais que caracterizam o inercialismo é volumosa e abrange um longo intervalo de tempo. Por isso, este artigo expôs da mesma apenas uma amostra, porém suficiente para mostrar que uma numerosa família de autores dos anos 50 a início dos anos 80 mostrou-se familiarizada com os conceitos básicos do inercialismo. 
Visto em detalhe, o inercialismo apresenta pelo menos duas correntes, uma extrema e outra moderada. A primeira é a de que a inflação presente depende exclusivamente da inflação passada, ou da inflação passada juntamente com choques inflacionários sobre os quais o governo não tem qualquer controle; a segunda diria que a taxa de inflação tem também outros determinantes, entre os quais políticas de demanda agregada e expectativas inflacionárias que olham para a frente. Os elementos principais e característicos de ambas as correntes são comuns, e, como se viu, reproduzem idéias correntes na literatura internacional dos anos 50 a 70 sobre inflação.

Há, porém, uma importante diferença de ênfase entre o inercialismo radical e suas raizes intelectuais do passado. Os economistas dos anos $60 \mathrm{e}$ 70 (inclusive Simonsen, no Brasil) entendiam a dependência exclusiva dos preços a preços passados como um caso limite de interesse apenas teórico. Eles interpretavam a evidência de regressões e de casos históricos de inflação como mostrando que a maior ou menor realimentação de preços provocava um atraso maior ou menor do impacto das políticas de demanda, mas sem negar que mais cedo ou mais tarde essas políticas tinham forte influência sobre a taxa de inflação. Os inercialistas radicais, em contraste, levaram a sério a fórmula de dependência exclusiva de preços a preços como a descrição adequada do processo inflacionário. Uma implicação importante deste modo de ver é que a inflação está completamente isolada de pressões de demanda.

O inercialismo radical prevaleceu no Brasil, sobretudo na fase de preparação e administração do Plano Cruzado; depois disso caiu em declínio, mas transformou-se na versão popular do inercialismo, aquela que aparece em notícias e comentários de jornais, bem como aquela a que, inadvertidamente, alguns inercialistas sofisticados eventualmente recorreram em comentários breves e em entrevistas.

\section{Referências bibliográficas}

ACKLEY, Gardner. Macroeconomic Theory. New York: Macmillan, 1961.

BAER, Werner. The Brazilian Economy: Its Growth and Development. Columbus: Grid, 1979.

BAILY, Martin. Contract Theory and the Moderation of Inflation by Recession and

by Controls. Brookings Papers on Economic Activity, no. 3, p. 585-633, 1976.

BLANCHARD, Olivier. Price Asynchronization and Price Level Inertia. Em:

DORNBUSCH, Rudiger; SIMONSEN, Mario. (orgs.). Inflation, Debt, and Indexation. Cambridge (MA): MIT Press, p. 3-24, 1983.

BLYTH, Conrad. Inflation in New Zealand. Sidney: George Allen \& Unwin, 1977. CROUCH, Robert. Macroeconomics, New York: Harcourt Brace Jovanovich, 1972. 
DICKS-MIREAUX, E. 1961. The Interretationship between Cost and Price Changes, 1946-1959: A Study of Inflation in Postwar Britain. Oxford Economic Papers, vol. 13, no. 3, p. 267-292, Oct: 1961 .

DORNBUSCH, Rudiger; FISCHER, Stanley. Budget Deficits and Inflation. Em: FLANDERS, M. \& RAZIN, Assaf (orgs.). Development in an Inflationary World. New York: Academic Press, 1981, p. 319-342.

DUESENBERRY, James. The Mechanics of Inflation. Review of Economics and Statistics, vol. 32, no. 2, p. 144-149, May 1950.

ECKSTEIN. Otto. Core Inflation. Englewood Cliffs:Prentice-Hall, 1981.

GUITTON, Henri. La Monnaie. Troisième édition, Paris: Dalloz, 1974.

GYLFASON, Thorvaldur \& LINDBECK, Assar. The Political Economy of Cost Inflation. Kyklos, vol. 35, no. 3, p. 430-455, 1982.

HAGGER, A. J. Inflation: Theory and Policy. London: MacMillan, 1977.

HOLZMAN, Franklyn. Income Determination in Open Inflation. Review of Economics and Statistics, vol. 32, no. 2, May 1950, p. 145-158.

JOHNSON, Harry. A Survey of Theories of Inflation. 1963. Reproduzido em JOHNSON, H. Essays in Monetary Economics. 2nd ed., London: George Allen and Unwin, 1969, cap. 3, p. 104-142.

KEISER, Norman. Macroeconomics. New York: Random House, 1971.

LEMGRUBER, Antônio C. Inflation in Brazil. Em: KRAUSE, Lawrence \& SALANT, Walter (orgs), Worldwide Inflation. Washington (DC): Brookings Institution, 1977.

LINDAUER, John. Macroeconomics. 2nd edition, New York: John Wiley \& Sons, 1971.

LUCAS, Robert E, Jr.; RAPPING, Leonard. Price Expectations and the Phillips Curve. American Economic Review, vol. 59, no. 3, p. 342-350, June 1969.

MAKINEN, Gail. Money, the Price Level, and Interest Rates. Englewood Cliffs: Prentice-Hall, 1977.

MEADE, James. The Intelligent Radical's Guide to Economic Policy. London: George Allen \& Unwin, 1975.

NORDHAUS, William. Inflation Theory and Policy. American Economic Review, vol. 66, no.2, p. 59-64, May 1976.

Macroconfusion: The Dilemmas of Economic Policy. Em: TOBIN, James (org.). Macroeconomics, Prices \& Quantities. Washington (DC): Brookings Institution, p. 247-284, 1983.

OKUN, Arthur. Inflation: Its Mechanisms and Welfare Costs. Brookings Papers on Economic Activity, no. 2, p. 351-401, 1975.

The Invisible Handshake and the Inflationary Process. Challenge, vol. 22, no. 6, p. 5-12, Jan.-Feb. 1980.

Prices and Quantities. Washington (DC): Brookings Institution, 1981.

PATINKIN, Don. The Inflationary Experience: Some Lessons from Israel. Em GAPINSKI, James; ROCKWOOD, Charles. Essays in Post-Keynesian Inflation. Cambridge (MA): Ballinger, cap. 7, p. 125-134, 1979.

PERRY, George. After the Freeze. Brookings Papers on Economic Activity, no. 2, p. 445-449, 1971.

Controls and Income Shares. Brookings Papers on Economic Activity, no. 1, p. 191-198, 1972 .

Determinants of Wage Inflation Around the World. Brookings Papers on Economic Activity, no. 2, p. 403-447, 1975. 
Slowing the Wage-Price Spiral: the Macroeconomic View. Brookings Papers on Economic Activity, no. 2, p. 259-299, 1978.

PHELPS, Edmund et al. Microeconomic Foundations of Employment and Inflation Theory. New York: W. W. Norton.

POHLMAN, Jerry. Inflation Under Control?. Reston: Reston, 1976.

ROWAN, D. C. Output, Inflation \& Growth. 2nd ed., London: Macmillan, 1974.

SCHULTZE, Charles. Recent Inflation in the United States. 1959. Reproduzido em

SHAPIRO, Edward (org.). Macroeconomics: Selected Readings. New York:

Harcourt, Brace \& World, cap. 25, p. 367-383, 1970.

SOBEL, Irwin. Abba Lerner on Employment and Inflation: A Post-Keynesian

Perspective. Em: GAPINSKI, James; ROCKWOOD, Charles. Essays in PostKeynesian Inflation. Cambridge (MA): Ballinger, cap. 15, p. 265-285, 1979.

TAYLOR, John B. Staggered Wage Setting in a Macro Model. American Economic Review, vol. 69, no. 2, p. 108-113, May 1979.

Aggregate Dynamics and Staggered Contracts. Journal of Political Economy, vol. 88, no. 1, p. 1-23, Feb. 1980.

TOBIN, James. There are Three Types of Inflation: We Have Two. 1974. Em: TOBIN, James. Policies for Prosperity. Cambridge (MA). MIT Press, cap. 28, p. 320-323, 1987.

Inflation: Monetary and Structural Causes and Cures. 1980. Em: TOBIN, James. Policies for Prosperity. Cambridge (MA): MIT Press, cap. 29, p. 324 $339,1987$.

Diagnosing Inflation: a Taxonomy. Em: FLANDERS, M. \& RAZIN, Assaf (orgs.). Development in an Inflationary World. New York: Academic Press, 1981 , p. 19-30.

Inflation. 1982. Em: TOBIN, James. Policies for Prosperity. Cambridge (MA): MIT Press, 1987. cap. 27, p. 301-319, 1987.

(org.). Macroeconomics, Prices \& Quantities. Washington (DC): Brookings Institution, 1983.

TREVITHICK, James; MULVEY, Charles. The Economics of Inflation. London: Martin Robertson, 1975.

TURVEY, Ralph. Counter-Inflationary Policies. 1978. Em Posner, Michael (org.). Demand Management. London: Heinemann, cap. 10, p. 191-199, 1978.

UNITED STATES GOVERNMENT. Economic Report of the President. Washington (DC): United States Government Printing Office, 1975.

VENIERIS, Yiannis; SEBOLD, Frederick. Macroeconomic Models and Policy, Santa Barbara: John Wiley \& Sons, 1977.

WACHTER, Michael. Some Problems in Wage Stabilization. American Economic Review, vol. 66, no. 2, p. 65-71, May 1976.

The Changing Cyclical Responsiveness of Wage Inflation. Brookings Papers on Economic Activity, no. 1, p. 115-167, 1976.

ZAWADZKI, K. The Economics of Inflationary Processes. London: Weidenfeld and Nicolson, 1965.

La Economía en los Procesos Inflacionários. Barcelona: Editorial Labor, 1974. 